\title{
A review on synchrophasor communication system: communication technologies, standards and applications
}

\author{
Bhargav Appasani ${ }^{1}$ and Dusmanta Kumar Mohanta ${ }^{2^{*}}$ (D)
}

\begin{abstract}
The present-day power system is a complex network that caters to the demands of several applications with diverse energy requirements. Such a complex network is susceptible to faults caused due to several reasons such as the failure of the equipment, hostile weather conditions, etc. These faults if not detected in the real-time may lead to cascading failures resulting in a blackout. These blackouts have catastrophic consequences which result in a huge loss of resources. For example, a blackout in 2004 caused an economic loss of 10 billion U.S dollars as per the report of the Electricity Consumers Resource Council. Subsequent investigation of the blackout revealed that the catastrophe could have been prevented if there was an early warning system. Similar other blackouts across the globe forced the power system engineers to devise an effective solution for real-time monitoring and control of the power system. The consequence of these efforts is the wide area measurement system (WAMS). The WAMS consists of several sensors known as the phasor measurement units (PMUs) that collect the real information pertaining to the health of the power grid. This information in the form time synchronized voltage and current phasors is communicated to the central control center or the phasor data concentrator (PDC) where the data is analyzed for detection of power system anomalies. The communication of the synchrophasor data from each PMU to the PDC constitutes the synchrophasor communication system (SPCS). Thus, the SPCS can be considered as the edifice of the WAMS and its reliable operation is essential for the effective monitoring and control of the power system. This paper presents a comprehensive review of the various synchrophasor communication technologies, communication standards and applications. It also identifies the existing knowledge gaps and the scope for future research work.
\end{abstract}

Keywords: Phasor measurement unit, Wide area measurement system, Synchrophasor communication system, Communication standards, Communication technologies, Optimal placement

\section{Introduction}

The modern-day power grid aims at providing reliable and quality power, which requires careful monitoring of the power grid against catastrophic faults [1]. The Phasor Measurement Unit (PMU) based Wide Area Measurement System (WAMS) was instituted for providing the time synchronized measurements pertaining to the health of the power system [2]. The timely detection of the faults and the subsequent contingency measures are not only dependent on the PMU but also

\footnotetext{
*Correspondence: dkmohanta@bitmesra.ac.in

${ }^{2}$ Department Electrical and Electronics Engineering, Birla Institute of

Technology, Mesra 835215, India

Full list of author information is available at the end of the article
}

on the underlying synchrophasor communication system (SPCS) [3]. It is analogous to the nervous system in the body. Just as the nervous system connects the various sensory organs to the brain and enables the interaction between them, the SPCS connects the PMUs to the Phasor Data Concentrator (PDC) or to the control center resulting in the exchange of data and control commands. Hence, the SPCS is considered as the edifice of the WAMS. The researchers have explored several PMU based monitoring and control schemes with the assumption that the underlying communication system is inherently capable of meeting the desired performance requirements. However, the subject matter of the SPCS is relatively less explored as compared with that of the 
PMU and this fact can be corroborated by a year wise comparative publication statistics illustrated in Fig. 1. It is observed that the literature on SPCS is very sparse and less than $10 \%$ of the published articles on PMU are related to the SPCS. In this regard, the chapter provides a comprehensive review of the various important pertaining to the SPCS that are available in the literature. These works are classified into the following topics and the topic-wise distribution of major works published in this context is shown in Fig. 2.

1. Synchrophasor communication standards.

2. Communication requirements for various WAMS applications.

3. Communication schemes for various WAMS applications.

4. Wide area control incorporating communication system parameters.

5. Reliability analysis of the SPCS.

6. Optimal placement of PMUs and their communication infrastructure.

7. Software-Defined Networking (SDNs) for PMUs

\subsection{Evolution of communication technologies in power system}

In the initial stages, the power system was very simple. The size of the system was small and the generating stations were mostly located nearby the load centers. The monitoring and control of the system were achieved based on the observation and judgment of the system operators. The control instructions were exchanged via verbal communication. By the mid of the 20th the size and complexity of the power systems increased enormously. The load centers were no longer located in the proximity of the generating stations and hence the need for communication systems for faster exchange of control information was realized. Supervisory control and

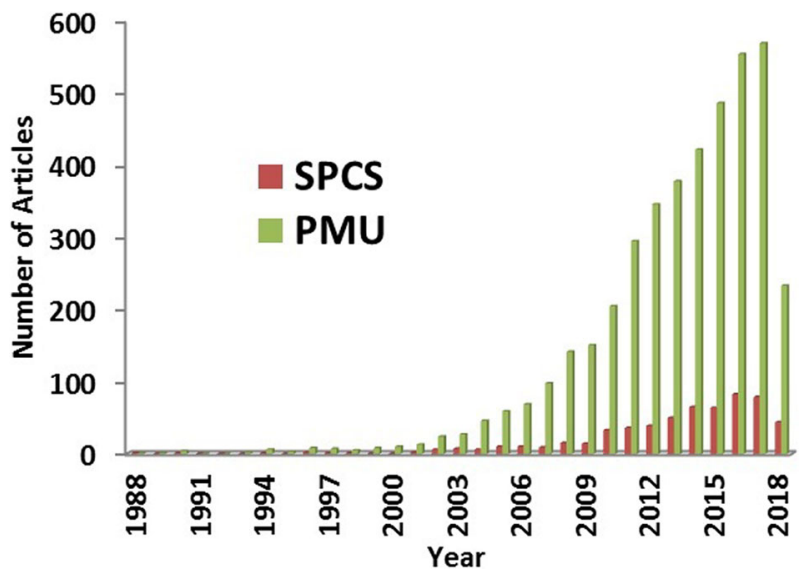

Fig. 1 Year-wise publication comparison data acquisition (SCADA) systems were developed to achieve the monitoring and control. Even at this stage, the number of stations was limited and hence telephone lines were primarily used for the purpose of data exchange [4].

However, with the increase in demand for electricity and the geographically distributed nature of the power system elements exacerbated the need for more sophisticated monitoring and control mechanisms. Huge amounts of data were to be measured at the load centers continuously and processed in real time to obtain meaningful interpretations of the health of the power system, thereby allowing the power system operator to execute appropriate control actions. Minicomputers and microprocessors were used for the purpose of obtaining the status regarding the power system. Powerline communication (PLC) systems were set up to enable the communication between the stations and the control centers. Information pertaining to the voltage, frequency, power flow, circuit breakers, isolators, etc. was measured at the station in cycles of every few seconds and was sent to the control center for continuous monitoring.

The synchronization of regional power grids led to the establishment of a national grid. The establishment of a national grid increased the complexity of the power system. In addition, the increase in the use of renewable sources of energy with their unpredictable nature presents a serious challenge for the monitoring of the system. In the early 80s PMUs were developed for effective real-time monitoring of such a large network. PMUs are capable of providing fine-grained measurements pertaining to the power system dynamics but they also require high speed, reliable and secure communication systems. These factors led to a drastic increase in the communication needs of the power system. The evolution of the communication needs in the power system for the purpose of monitoring and control goes hand in hand with the evolution of the measurement technology. The evolution of communication technologies in the power system is depicted in Fig. 3 [4].

\subsection{Functions of a communication network in a power system}

The important functions of communication networks in a power system are listed below.

(a) The most important function of the communication system is to facilitate the real-time detection of faults and to facilitate the implementation of the appropriate control actions thereby ensuring the stable operation of the power system.

(b) Optimization of the power flow through real-time economic load dispatch. 


\section{- Synchrophasor \\ Communication Standards \\ a Communication requirements for WAMS applications \\ novel Communication Schemes \\ - Wide Area Control Incorporating Communication Parameters \\ neliability Analysis pf SPCS \\ Optimal Placement of SPCS \\ - Software Defined Networking for PMUs}

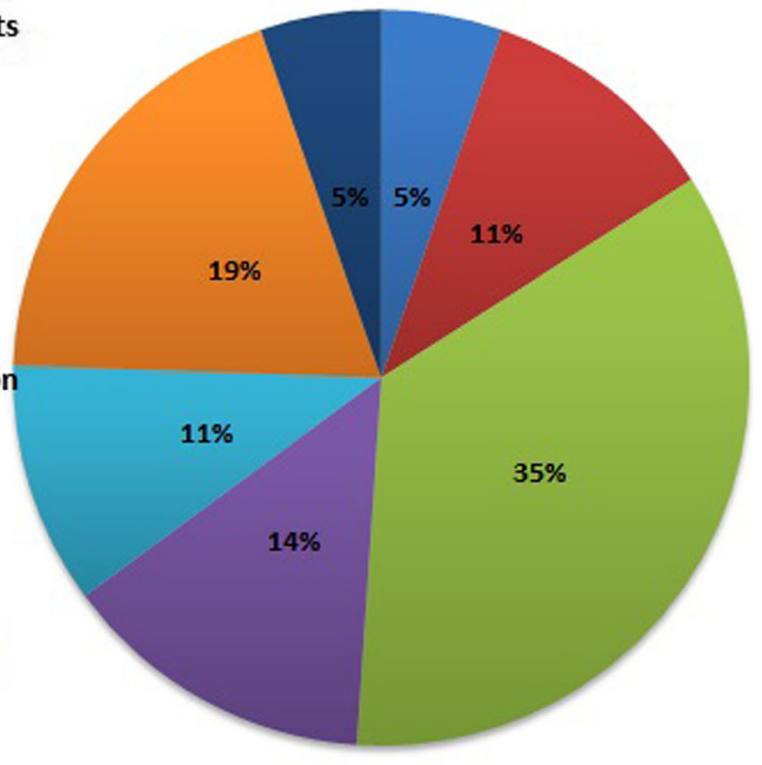

Fig. 2 Topic-wise distribution of literature on SPCS

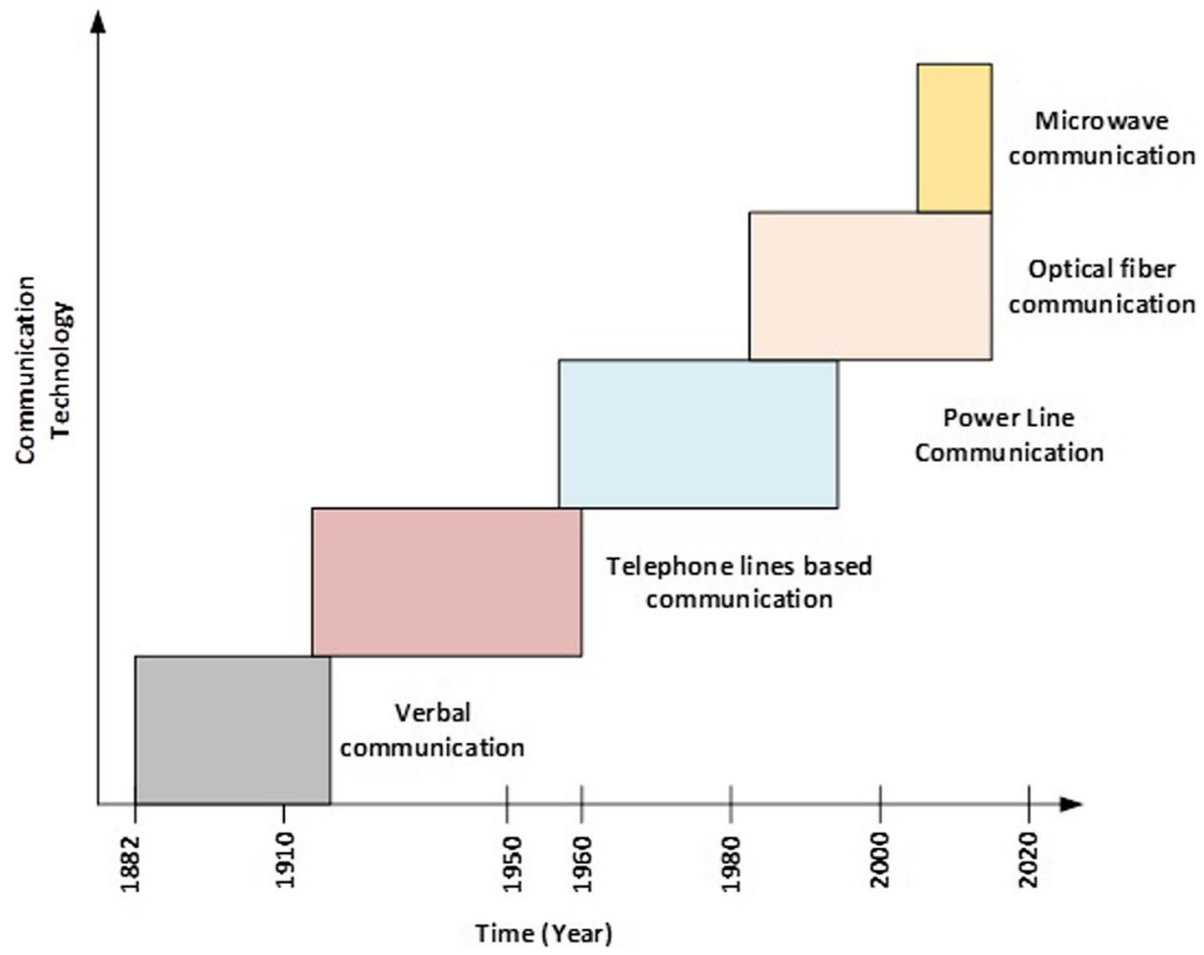

Fig. 3 Evolution of communication technologies in power system 
(c) Wide area protection schemes for the various generating sources and transmission infrastructure.

Security and reliability are the most important requirements of a communication system. The data flowing through the communication system should be inaccessible to unauthorized agents and the failure rate of the communication infrastructure should be as low as possible in order to facilitate the implementation of real-time monitoring and control actions.

\subsection{Organization of the paper}

This paper presents a comprehensive review of the communication system for synchrophasor applications and is organized into five sections. To better appreciate the importance of the SPCS a brief description of the WAMS is given in the second section. This section also describes the various synchrophasor communication technologies. The synchrophasor communication standards are described in the third section. All the PMUs and the PDCs manufactured by various vendors have to comply with these standards for the exchange of data.

The communication network architecture, the location of the infrastructure and the quality of service has to be assessed for effectual operation of the WAMS. Researchers have proposed network architectures and have created simulation models for evaluating the performance of the practical SPCS. These various contributions are reviewed in the fourth section. The other aspects of SPCS that have received the attention of the researchers are the optimal placement of the PMU communication infrastructure and software-defined networking for PMUs. These works are separately discussed in the fifth and sixth sections respectively. The last section identifies the knowledge gaps and the potential challenges that can act as an inspiration for future research.

\section{A brief description of the WAMS}

In the past few decades, the PMU has carved its niche as an important sensor for estimating the state of the power system [5]. The PMUs are installed at the electrical nodes, which are usually separated by large geographical distances. The measurements from several such PMUs are reported to the local PDC. Data from several such local PDCs may be passed on to a regional PDC which is on a higher level in the hierarchy. The PMUs, the PDCs and the SPCS together constitute the WAMS and a typical WAMS is illustrated in Fig. 4.

The IEEE defines the PMU as "a device that produces synchronized phasor, frequency, and rate of change of frequency (ROCOF) estimates from voltage and/or current signals and a time synchronizing signal". Thus, the PMU is a digital signal processing unit that can

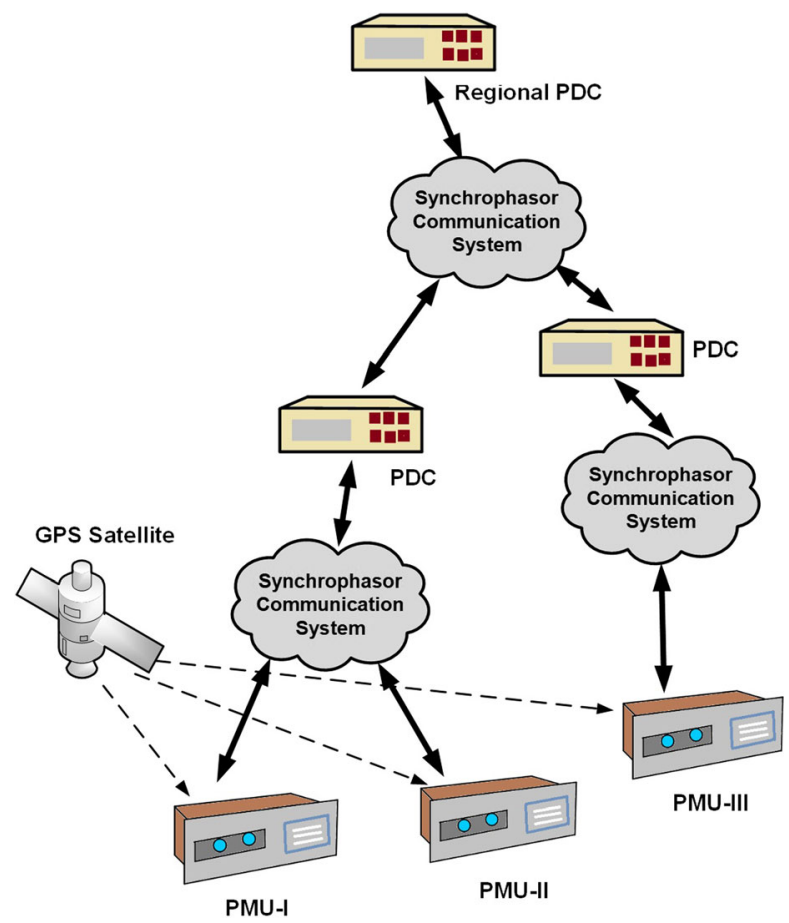

Fig. 4 A typical WAMS systems with communication hierarchy

calculate the voltage phasor and current phasor, time synchronizes these phasors and communicates them to the PDC. Time synchronization is achieved with the aid of the timing signals received from the Global Positioning System (GPS) satellites. The various constituent components of the PMU are depicted in the block diagram illustrated in Fig. 5 [6].

The current and potential transformers located in the power system network provide the current and voltage measurements to the PMU in the form of analog inputs required for digital processing. To prevent the aliasing of the sampled signals, the inputs are fed through an anti-aliasing filter that limits the bandwidth of the input analog signal as per the requirements. The output of the anti-aliasing filter is further fed to an analog to digital (A/D) converter for converting it into the desired digital output which is fed into a central processing unit (CPU). The CPU computes the magnitude and the phase of the input signal using the DFT technique and time synchronizes the data using a sampling clock that is phase locked to the one-pulse-per-second provided by the GPS receiver. As the signal is time stamped, it is transmitted from the PMU to the PDC through the synchrophasor communication network.

The key communication technologies, for synchrophasor data transfer, can be broadly classified into two categories: wired and wireless [7]. The wired communication technology offers high reliability, huge bandwidth and protection against interference $[8,9]$. On the other hand, 


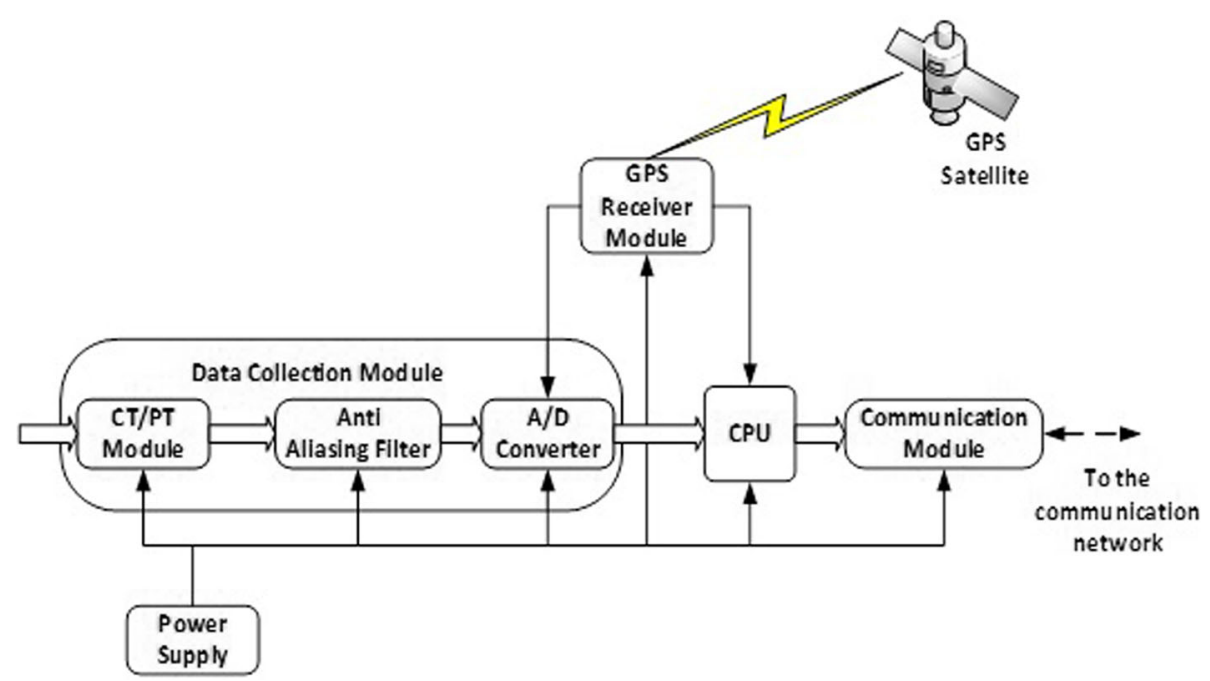

Fig. 5 The various functional blocks in the PMU

wireless technology enjoys superiority in terms of rapid deployment, low installation and maintenance costs, access to remote geographic locations, etc. The various synchrophasor communication technologies are depicted in Fig. 6.

\subsection{Power line communication for Synchrophasor data transfer}

This power line communication (PLC) uses the existing power line cables for the transfer of synchrophasor data [10]. As there is no need for additional communication infrastructure, this technology provides the fastest and economical means for the deployment of communication networks. There are two types of PLC technologies: Narrow Band PLC (NB-PLC), which is for low bandwidth applications and Broad Band PLC (BB-PLC), which is for applications requiring higher bandwidth. As the synchrophasor application is mission critical, BB-PLC would be the preferred choice. Typical data rates ranging from 2 to 3 Mbps can be achieved using this technology. The use of this communication technology for synchrophasor applications can be understood from Fig. 7 .

In spite of having several advantages, the technology suffers from many disadvantages such as the difficulty in modeling the communication channel due to the noisy background in the power cables. Moreover, the PLC is not meant for high bandwidth applications due to fading and interference. The signal to noise ratio (SNR) is low and this technology is usually combined with other communication technologies, such as cellular communication, to provide a hybrid solution for power grid communications.

\subsection{Optical Fiber-based Synchrophasor communication system}

The benefits of optical fibers for long-distance communication has been known to the communication engineers since the early 1990s. The practical use of these fibers for communication gained momentum in the early 2000s due to a substantial reduction in the cost of the cables and improvement in their quality. The high data rates, low attenuation, high reliability and negligible interference

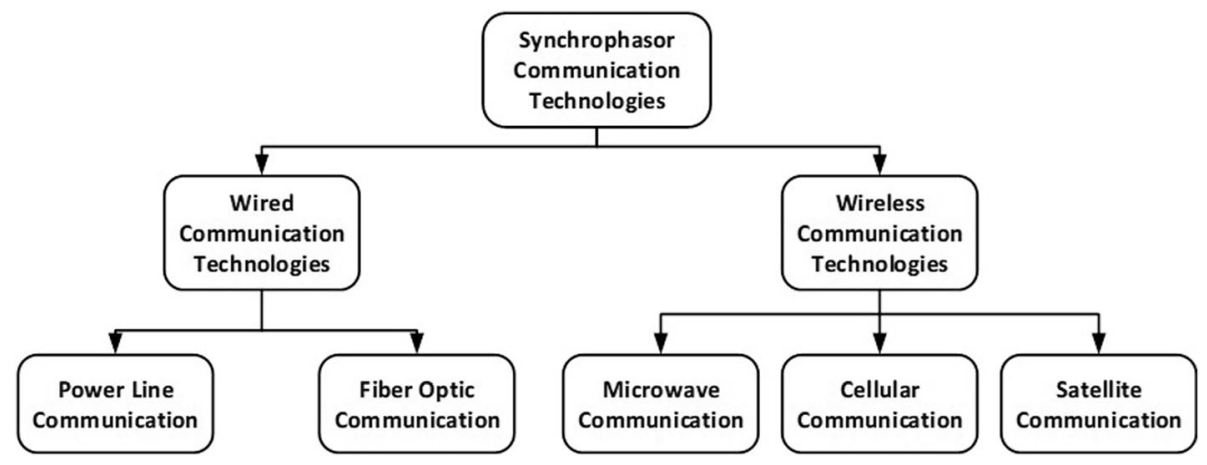

Fig. 6 The various synchrophasor communication technologies 


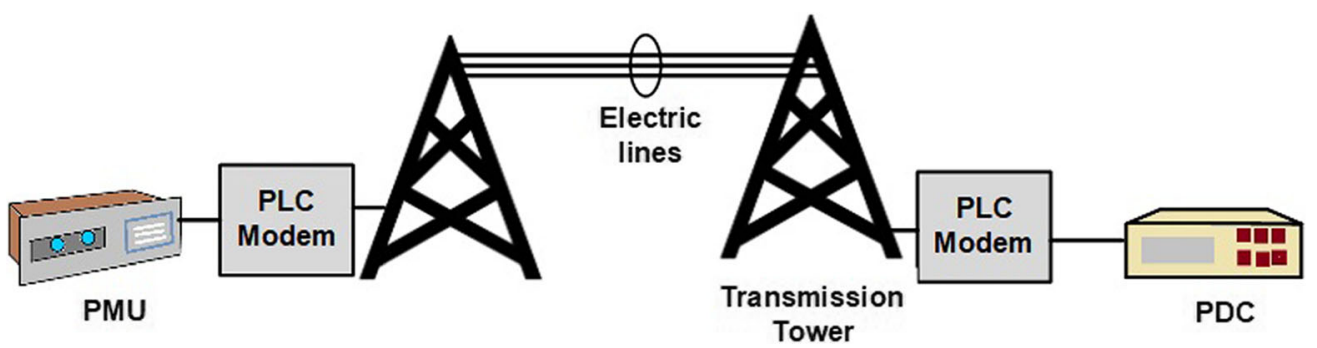

Fig. 7 PLC for Synchrophasor Data Transfer

made them a widely used synchrophasor communication technology [11]. They also have applications in substation automation, tele-protection, tele-control, vehicle to grid communications, etc. A typical fiber optic SPCS is shown in Fig. 8.

The data received from the PMU is converted into an optical signal by the optical transmitter, which is basically a light emitting diode or a laser. These optical signals are then carried via the optical fibers to the PDC. At the PDC, the optical signal is converted back into electrical signals using a photodiode. Between the PMU and the PDC, optical repeaters are placed at regular intervals to boost the signal strength and to maintain the signal quality.

This technology even though widely adopted for synchrophasor data transfer is still plagued by several problems that are common to the other wired communication technologies. The installation cost is very high and substantial time is required to lay and erect the communication infrastructure. Moreover, it is also difficult to install the cables in hilly or rocky terrain.

\subsection{Cellular communication for Synchrophasor data transfer}

Due to the high proliferation of the wireless communication infrastructure, cellular communication technology provides another viable alternative for synchrophasor data transfer [11, 12]. It is one of the fastest growing technologies in the world and the research is already in progress to achieve data rates of $100 \mathrm{Gbps}$ per user. The different cellular technologies that can cater to the demands of the synchrophasor application are shown in Table 1. Even though this technology can cater to the demands of the synchrophasor application in terms of the data rate requirements, the shared nature of this technology makes it unacceptable for mission-critical applications that require uninterrupted communication services. The cellular technology based SPCS is illustrated in Fig. 9.

2.4 Satellite communication for Synchrophasor data transfer Satellite communication technology has undergone tremendous growth over the past few decades. The uniqueness of this technology is that the communication equipment is located in space. Thus, this technology is unaffected by natural disasters such as floods, earthquakes, etc. It consists of two different segments: the earth segment and the space segment. The space segment consists of the satellites and the ground facilities that are responsible for Tracking Telemetry and Control (TT\&C). The earth segment consists of transmitting and receiving earth stations. This technology can be used for the transfer of synchrophasor data when the end equipment is separated by several hundred kilometers. However, the major disadvantage for synchrophasor application is that communication delay is higher compared to any other technology and is seldom used [12]. The satellite-based SPCS is shown in Fig. 10.

\subsection{Microwave-based Synchrophasor communication system} Microwave communication is another wireless communication technology that can cater to the demands of the synchrophasor applications [12]. With the growth of wireless technology, data rates in the order of several Gbps are being achieved. Thus, this technology provides a viable alternative to the optical fiber SPCS, as it has

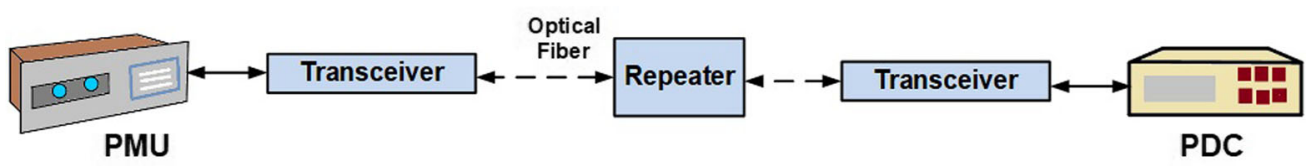

Fig. 8 Optical Fiber Synchrophasor Communication System 
Table 1 Cellular Technologies for Synchrophasor Applications

\begin{tabular}{ll}
\hline Technology & Data Rates \\
\hline General Packet Radio Service (GPRS) & Up to $114 \mathrm{Kbps}$ \\
$\begin{array}{l}\text { Enhanced Data rates for GSM Evolution } \\
\text { (EDGE) }\end{array}$ & Up to $384 \mathrm{Kbps}$ \\
$\begin{array}{l}\text { Universal Mobile Telecommunications } \\
\text { System (UMTS) }\end{array}$ & Upto $2 \mathrm{Mbps}$ \\
High-Speed Packet Access (HSPA) & $600 \mathrm{Kbps}-10 \mathrm{Mbps}$ \\
Long Term Evolution-Advanced (LTE-A) & Up to $100 \mathrm{Mbps}$ \\
\hline
\end{tabular}

several conspicuous advantages which have been presented in section 1.3 of the first chapter. A typical microwave based SPCS is shown in Fig. 11.

In the microwave communication, the synchrophasor data generated by the PMU is fed to a PMU radio terminal that converts the electrical signal into radio frequency (RF) signal. The RF signal is then fed to a microwave antenna that converts the RF signal into an electromagnetic signal. This electromagnetic signal propagates in free space and is received by the microwave antenna at the PDC. The PDC radio terminal converts the RF signal back into the electrical signal, which is sent to the PDC for analysis. Intermediate repeaters are required to maintain the signal strength and to ensure communication feasibility in the case of non-line of sight propagation. The main disadvantage of this technology is that the signal propagates in free space and hence is susceptible to cyber-physical attacks.

The PDC can exchange the data and commands with PMUs and also with other PDCs. It receives data from multiple PMUs, performs a check on the received data, time aligns the data and creates an output stream. It may also provide the power system operator with appropriate visualizations that are needed for monitoring and control.

\section{Review of the Synchrophasor communication standards}

To standardize the communication of synchrophasors between the devices manufactured by different vendors, IEEE has developed a synchrophasor data transfer standard. This standard defines the method for exchange of synchrophasor data between the PMUs, the PDC and other applications. The initial standard for the synchrophasor measurement was the IEEE Std 1344-1995 [13]. This standard was reaffirmed in the year 2001 and replaced by the IEEE Std C37.118-2005 in the year 2005 [14]. The IEEE Std C37.118-2005 introduced the concepts of compliance testing and Total Vector Error (TVE). It also provided clarification regarding the definitions of the phasor and synchrophasor. The message formats were revised to enhance the exchange of information with other systems. Additional fields were added to the message frames and the existing format was replaced to improve the information exchange. This standard specified both the communication requirements and the measurement requirements. To increase the widespread adoption of this standard, IEEE further split this into two standards: IEEE Std C37.118.1 [15, 16] which defines the synchrophasor measurement requirements and the IEEE Std C37.118.2-2011 [17, 18] which defines the synchrophasor communication requirements. The IEEE Std C.37.118.2-2011 contains all the communication requirements specified in its predecessor standard and an additional configuration frame has been incorporated. The IEEE Std C37.118.2-2011 specifies the message formats for enabling the transfer of synchrophasor data. The

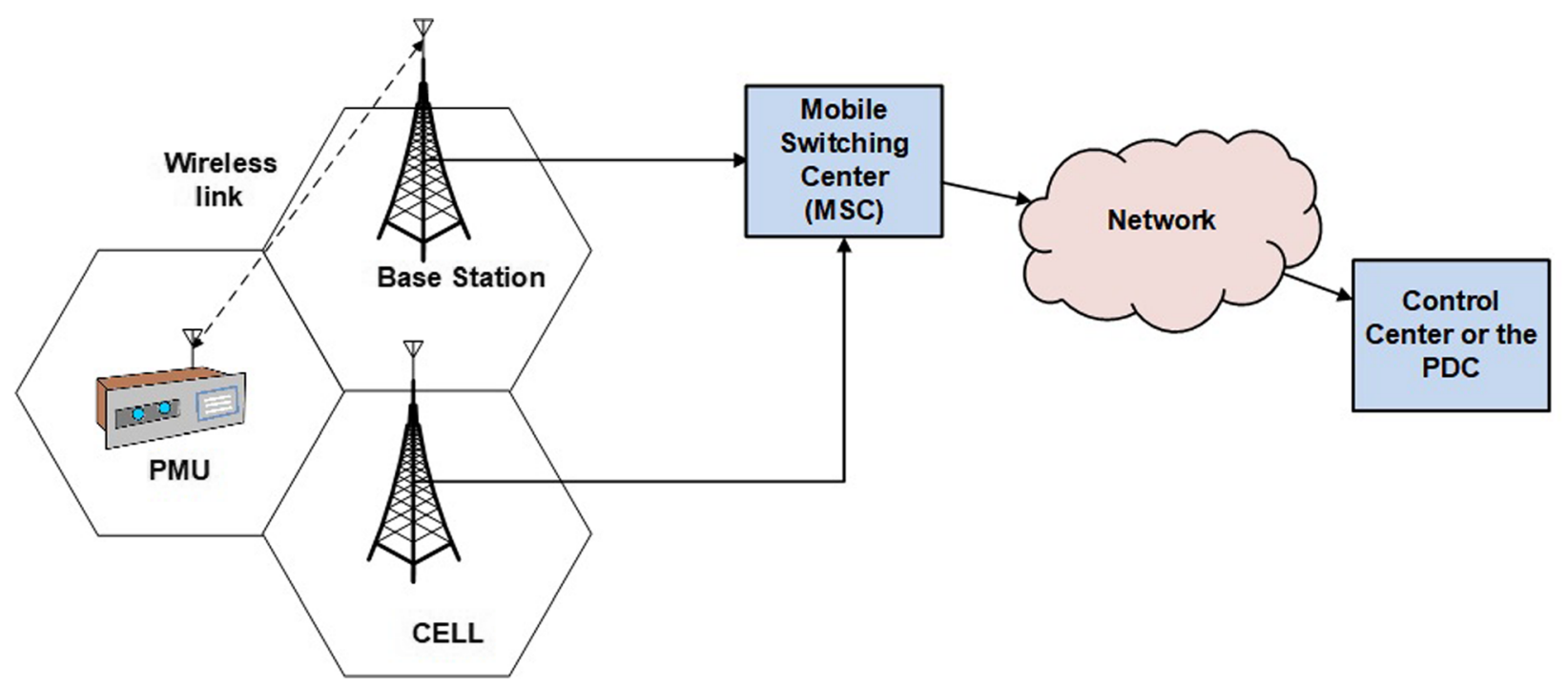

Fig. 9 Cellular Technology Based Synchrophasor Communication System 


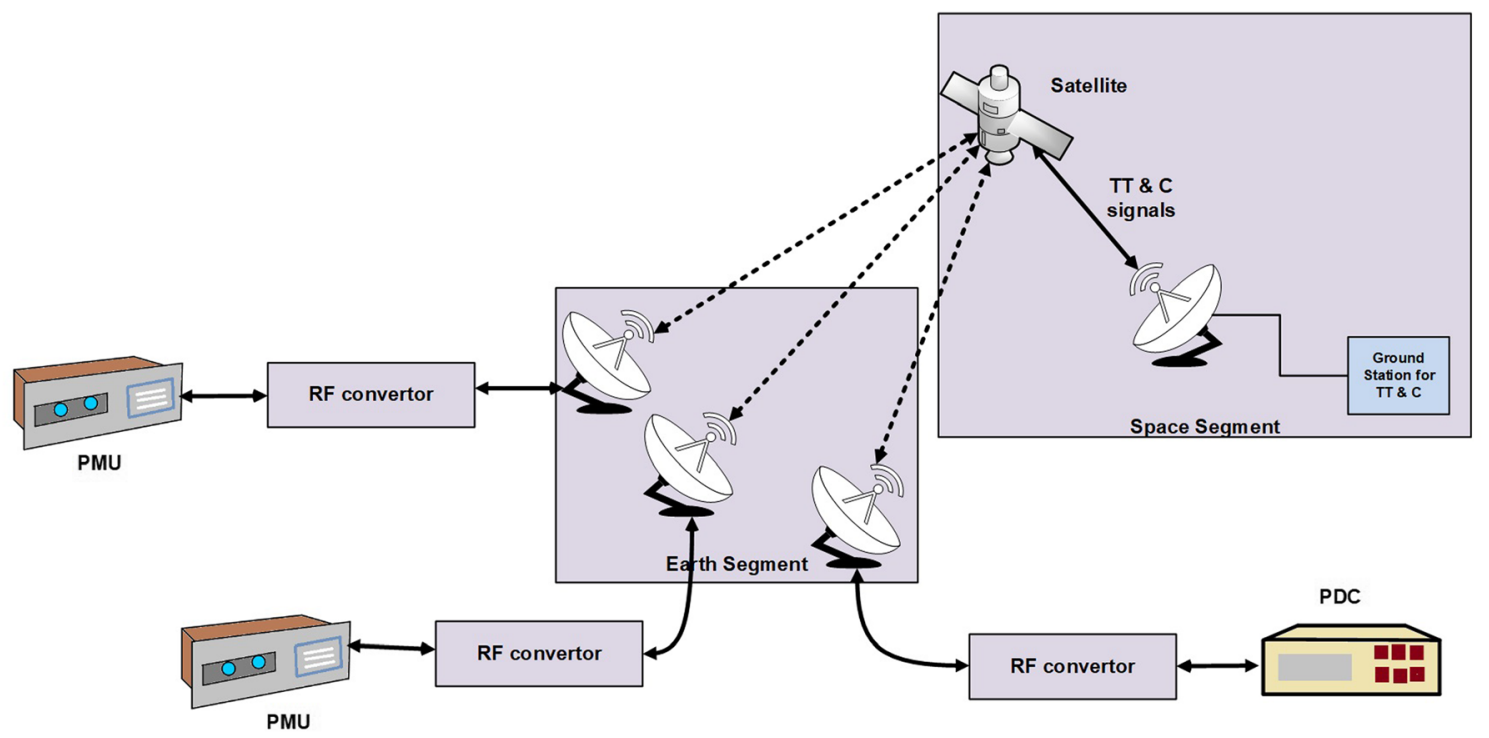

Fig. 10 Satellite-Based Synchrophasor Communication System

document is conceived in six separate clauses, which are summarized below:

Clause 1: Scope and need for the standard

Clause 2: References to the other standards related to the current standard

Clause 3: Defines the terminology found in the standard

Clause 4: Presents the background for synchronized phasor measurements

Clause 5: Describes the synchrophasor measurement system

Clause 6: Describes the communication protocol and message formats

The IEEE C37.118.2-2011 standard defines the message format for the transfer of data between the PMU and the PDC. The various fields of the message frame are shown in Fig. 12 and are briefly described in Table 2.

There are four types of message frames that are exchanged between the PMU and the PDC. These are data,

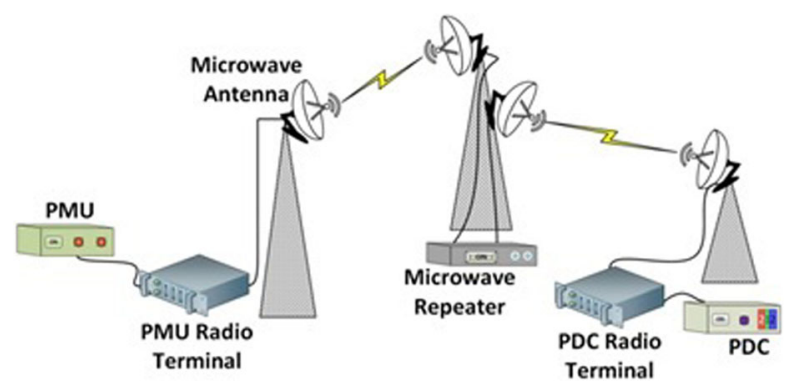

Fig. 11 Microwave Based Synchrophasor Communication System configuration, header and command frames. The first three frames, namely, the data frame, the configuration frame and the configuration frame are sent from the PMU to the PDC. The last frame is the command frame that sent from the PDC to the PMU. A summary of each of these frames is presented in Table 3.

\section{Review of the Synchrophasor communication requirements, communication schemes and their applications}

The SPCS consists of equipment that is spread over a large geographical sprawl. Modeling of the SPCS and their subsequent analysis through simulations can provide crucial insights into their performance prior to their physical deployment. A considerable amount of research has gone into areas such as identifying the communication requirements, modeling of the communication networks, analyzing their performance through simulations and developing schemes for enhancing the real-time control of the power system.

\subsection{Communication requirements for various WAMS applications}

Synchrophasor technology has several applications with varied communication requirements. State Estimation (SE), intelligent load shedding, islanding, wide area System Integration Protection Schemes (SIPS), oscillation

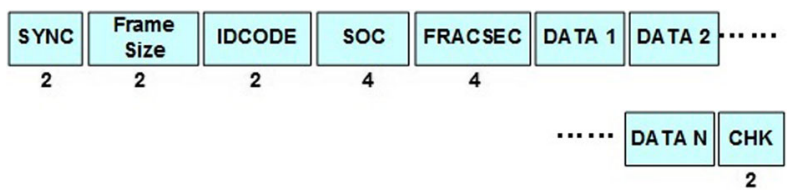

Fig. 12 The Various Fields of the Synchrophasor Message Frame 
Table 2 Description of the Various Fields of the Message Frame

\begin{tabular}{lll}
\hline Field & Size (Bytes) & Description \\
\hline SYNC & 2 & $\begin{array}{l}\text { This field marks the beginning of the frame. The first byte is AA (Hex) and the next byte describes the type } \\
\text { of the message. }\end{array}$ \\
$\begin{array}{lll}\text { Frame Size } & 2 & \begin{array}{l}\text { This field presents the length of the entire message including the CHK field. } \\
\text { This field is used for identifying the data stream. }\end{array} \\
\text { IDCODE } & 2 & \begin{array}{l}\text { This field carries the information about the time stamp and is used for time synchronization of measurements. } \\
\text { This field indicates the time at which the measurements are made for data messages and the time at which } \\
\text { the frame is transmitted for other messages. }\end{array} \\
\text { CHK } & 4 & \text { This field marks the end of the message frame and is used to perform the cyclic redundancy check. }\end{array}$
\end{tabular}

control, synchronization, etc. are some of the applications based on synchrophasor technology. The communication requirements for these applications have been theoretically analyzed and are summarized in Table 4 [19]. Further theoretical studies were carried out by Lin et al. to identify the interdependency between the power system applications and the communication infrastructure [20] and, a probabilistic model-based approach was proposed in [21] to obtain the communication delay characteristics in a WAMS. However, these theoretical studies have not been verified through suitable simulation models [22]. Simulation results can provide a realistic perception of the operation of practical communication networks.

Performance considerations for various synchrophasor applications have been identified by Chenine et al. through suitable simulations [23]. They also developed simulation models for the Nordic region and identified the communication requirements in terms of bandwidth and delay [24]. These models consider the communication networks to be dedicated to carrying only the synchrophasor data. However, in practical networks, the communication resources are shared and background traffic exists, which affects the performance of these applications $[25,26]$. The dedicated and shared communication networks for synchrophasor applications are shown in Fig. 13.

In dedicated networks, the communication resources are entirely utilized for synchrophasor communication and no other application can utilize these resources. Thus, the source of delay would be the processing delay at the PMU and the propagation delay. Thus, dedicated networks can be used to realize all of the synchrophasor application. Shared networks are economical and can be rapidly deployed. The communication resources are shared by several applications which increases the end-to-end delay and reduces the reliability of the communication network. As the synchrophasor applications are mission-critical, it is desirable to use dedicated communication resources, even though it increases the overall cost of the system.

The performance of the communication networks also depends on the transport layer protocol. The effect of these protocols on the delay characteristics has been carried out by Babazadeh et al. [27] and, in particular, an in-depth study of the communication delay in TCP/IP networks was carried out in [28-30]. TCP/IP is connection-oriented protocol that involves handshaking between the communicating devices. The presence of handshaking reduces the packet loss ratio but increases the end-to-end devices. Synchrophasor applications that require low communication latency should use connectionless protocols like UDP. Using connectionless protocols reduces the communication delay as there is no concept of handshaking. However, this increases the packet loss thereby reducing the reliability. Thus, the choice between reliability and latency must be carefully balanced and the selection of the transport layer protocol should be based on the application requirement.

Table 3 Summary of the IEEE C37.118.2-2011 message frames

\begin{tabular}{ll}
\hline Frame Type & Function \\
\hline Data frame & $\begin{array}{l}\text { This contains the real-time synchrophasor data measured by the PMU. It includes an identification header, } \\
\text { the length of the message, message source ID, a time stamp, detailed status information regarding the } \\
\text { data and its source and quality, frequency, ROCOF and analog and digital quantities. }\end{array}$
\end{tabular}

Header frame

Configuration frame
The header frame is sent from the PMU to the PDC to help the PDC identify the sender.

There are three configuration frames. The first configuration frame config 1 indicates the data reporting capability of the PMU. The config 2 and config 3 indicate the current measurements that are being reported in the data frame.

These frames are sent by the data receiving device to the data transmitting device requesting it to start or stop the transmission, to transmit the configuration frame or the header frame. 
Table 4 Communication Requirements for WAMS Applications

\begin{tabular}{lll}
\hline Application & Communication Delay (msec) & Data Rates \\
\hline State Estimation & 100 & $136.8 \mathrm{Kbps}$ \\
Generator Synchronization & 50 & $91.2 \mathrm{Kbps}$ \\
Intelligent Scheduling & 50 & $300 \mathrm{Kbps}$ \\
Islanding & 50 & $10 \mathrm{Kbps}$ \\
Oscillation control & 200 & $27.4 \mathrm{Kbps}$ \\
\hline
\end{tabular}

4.2 Review of the various Synchrophasor communication schemes and communication architectures

Synchrophasor communication networks for various applications have been proposed in the literature. Communication schemes based on TCP/IP [28, 29, 31], Controller Area Network (CAN) bus [32, 33], 3G [34, 35], LTE [36], optical fiber [37-39], Internet of Things (IoT) [40], Wireless Local Area Networks (WLAN) [41] etc. have been proposed. Apart from these simple communication schemes several other novel methods have been reported in the literature which are summarized in Table 5 .

The communication architecture also affects the performance of the WAMS. To analyze this effect Deng and his colleagues have modeled various wide area measurement scenarios and obtained the simulation results. They have observed that most WAMS applications can perform satisfactorily by properly choosing the network architecture and communication protocol [41]. There are two types of network architectures: centralized networks and decentralized or distributed networks. These two architectures differ in terms of network latency, reliability and cost and are shown in Fig. 14.

In the centralized architecture the PMUs report to a single control center. This architecture requires simple communication strategies but it is less reliable than the decentralized architecture especially for large power systems. The failure of communication networks would seriously affect the monitoring capability of the WAMS.

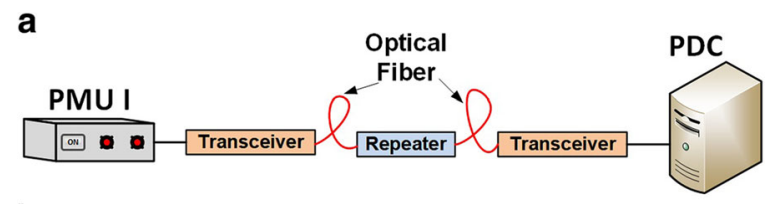

b

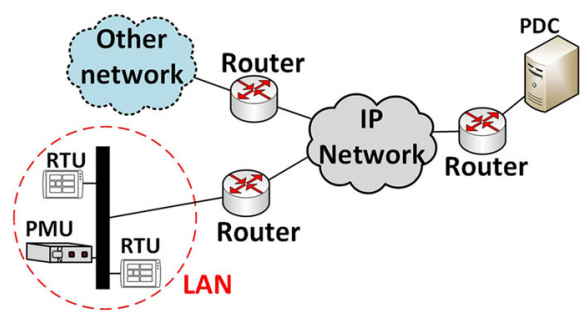

Fig. 13 a Dedicated Communication Network. b Shared Communication Network
On the other hand, in the distributed architecture there are several control centers that can coordinate among themselves in implementing the control action. Such networks are more robust to component failures but are more expensive and the communication requirements are complicated. Depending on the WAMS application, the size of the system and the communication requirements, both the network architectures have been incorporated and analyzed by the researchers.

A study on the communication in decentralized networks has been carried out in [42]. This work discusses communication grouping and routing problems in large networks. A decentralized control approach was proposed in [43], where the communication amongst the control centers was implemented using the messagepassing interface (MPI) protocol for obtaining the state of the wide area power system. Another novel distributed phasor measurement system and its communication networks were analyzed in [44] for obtaining the dynamic state of the power system. In [45], three different communication architectures were proposed for a decentralized control application where the local control centers can share their estimates obtained from the local PMUs with other control centers to compute the global estimate of the system. These architectures were analyzed for their relative merit with the aid of simulations carried on IEEE 68 bus and IEEE 145 bus systems.

Chenine and Nordström have modeled the centralized communication architecture and obtained the simulation results for various wide area measurement scenarios [46]. Centralized communication architecture has been considered in [47] for obtaining the communication delays in WAMS with the PMUs spread over large geographical separations. A co-simulator has been developed in [48] for simultaneously simulating the power system along with the communication infrastructure. Several communication network architectures have been modeled and simulated using this hybrid approach. Three different communication architectures have been proposed and verified for their relative merits and de-merits through suitable simulations. Another co-simulation platform was proposed in [49] for emulating the power system as well as the communication networks. Using this platform, the impact of communication delays, packet losses and noise in the data on the monitoring and control capabilities of the WAMS was investigated.

\subsection{Review of the various methods for wide area control incorporating communication parameters}

Another domain that has attracted the attention of the power system engineers is the use of PMU measurements for damping of oscillations. These wide area damping controllers are susceptible to communication delays and packet losses. Several works have been 
Table 5 Synchrophasor Communication Schemes

\begin{tabular}{ll}
\hline Reference & Contribution \\
\hline [72] & A multi-protocol label switching aided IP with better performance than the TCP/IP and other transport layer protocols. \\
{$[73]$} & An evolutionary traffic routing algorithm for routing the traffic such that the communication delay is minimized. \\
{$[74]$} & An advanced TCP/IP protocol that minimizes the communication delay by reducing redundant transmissions in the event of packet loss \\
{$[75-77]$} & $\begin{array}{l}\text { An internet for synchrophasor applications called as NASPInet developed by the collaborated effort of U.S. Department of Energy (DoE), } \\
\text { North American Electric Reliability Corporation (NERC), North American electric utilities and other individuals from industry and academia. }\end{array}$ \\
{$[78]$} & $\begin{array}{l}\text { A co-operative congestion control protocol through coordinated scheduling and bandwidth reassignment for minimizing communication } \\
\text { delay and to ensure real-time data transfer. Congestion causes unnecessary packet losses and also increases the wait time. Therefore, }\end{array}$ \\
minimizing congestion effectively reduces the communication delay. \\
{$[79]$} & $\begin{array}{l}\text { A low power communication scheme for secure PMU communications. } \\
{[80]}\end{array} \quad \begin{array}{l}\text { Synchrophasor communication network for hydropower plants. } \\
{[81,82]} \\
\quad \text { Communication networks for generation, substation and control center have been modeled and the performance is evaluated in terms } \\
\text { of delay for various bandwidth criteria. }\end{array}$ \\
\hline
\end{tabular}

a

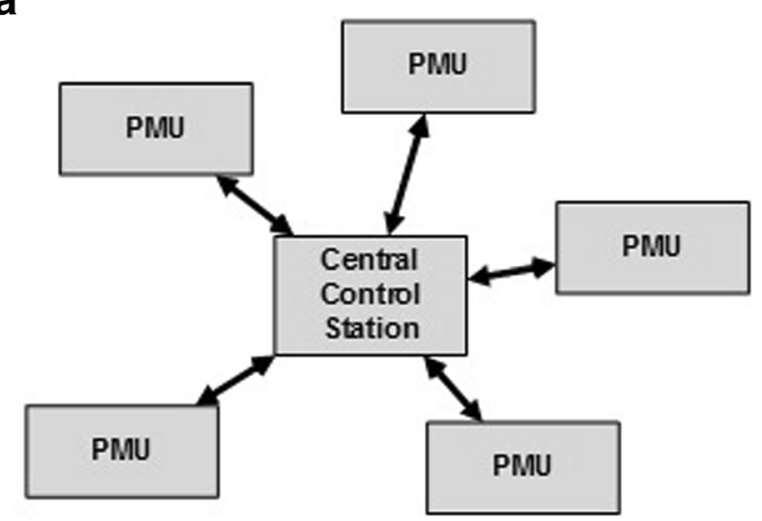

b

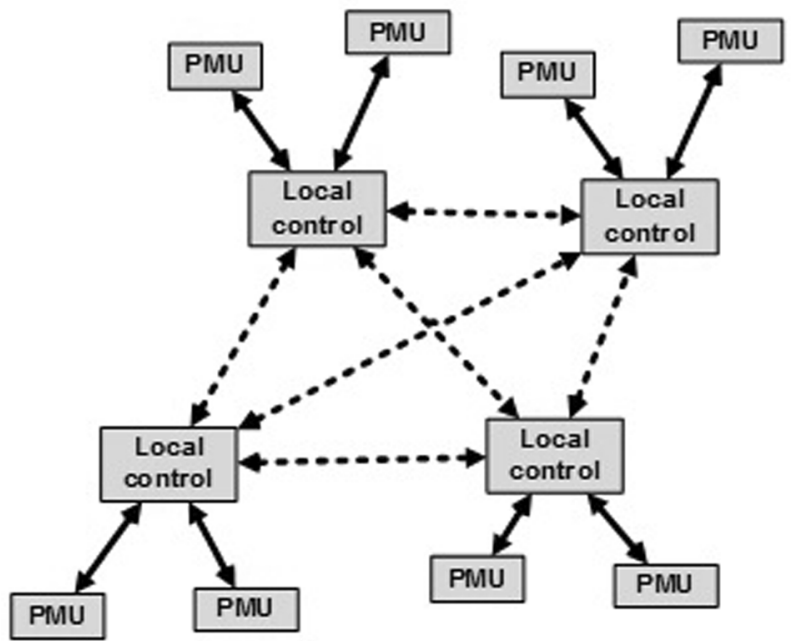

Fig. 14 a Centralized architecture. b Decentralized architecture proposed in this regard to achieve improved wide area control. The communication delay essentially causes a time-varying phase lag to the wide area damping system whereas packet losses results are scarce data being available for achieving wide area control. Moreover, the uncertainties in the system parameters pose a severe challenge for estimating the communication delay and the packet loss ratios. Thus, several works have been reported for modeling these losses and delays for realizing effective damping control of the power system. The important works in this regard are described in Table 6.

\section{Optimal placement of SPCS and their reliability analysis}

The reliable operation of the PMUs and their communication infrastructure is paramount for the safe operation of the grid. A state-of-the-art review of the various methods for reliability analysis of the PMUs is presented in [5]. However, the literature available on the reliability analysis of the SPCS is very sparse. Another research problem that has grabbed the attention of the researchers is the optimal placement of the PMUs and their communication infrastructure. As is the case with the reliability analysis, the problem of optimal placement of PMU (OPP) has been extensively explored by the researchers but the problem of optimal placement of the PMU communication networks has not been sufficiently explored.

\subsection{Reliability analysis of the SPCS}

The earliest work on the reliability analysis of the SPCS has been carried out by W. Liu and his colleagues [50]. They model the SPCS as an auxiliary evaluation system. Series-parallel networks are used for obtaining the reliability parameters. The proposed approach is used to obtain the reliability parameters of an IEEE 14 bus system. In [51], the WAMS was modeled as a combination of both the PMUs and the communication networks. The communication network reliability is measured in 
Table 6 Wide Area Damping Controllers

\begin{tabular}{ll}
\hline Reference & Significance \\
\hline [83-85] & Requirements for wide area control in terms of communication delay and data losses have been identified. \\
{$[45]$} & $\begin{array}{l}\text { A distributed multi-agent control concept for achieving wide area control. The local PMU measurements and local optimization } \\
\text { algorithms are shared to achieve global control. }\end{array}$ \\
{$[86]$} & Uncertainties in delays were included in the analysis and a centralized non-linear controller was used. \\
{$[87-90]$} & Communication delays were compensated to achieve the damping control. \\
{$[91]$} & A novel Flexible AC Transmission System (FACTS) controller architecture has been proposed to achieve improved control even \\
{$[92]$} & Ehen sufficient measurement data is available (due to bandwidth constraints or packet losses). \\
{$[93]$} & Adaptive PDC that configures itself based on communication delays for effective delay compensation of the wide area controller. \\
{$[94]$} & Damping of power system oscillations using a co-simulation platform for simulating the power and communication infrastructures. \\
{$[95]$} & Time delay estimate and packet loss probability were obtained using Markov models to achieve improved oscillation control. \\
\hline
\end{tabular}

terms of the intermediate node failures. A minimum path is considered between the source and destination pair and the reliability is measured in terms of the probability of connectivity existing between the nodes. The reliability parameters were obtained for the Henan power grid, China to substantiate the merit of the proposed approach. In [52], assessment of the effect of communication infrastructure failure on the various WAMS applications has been investigated. Even though the paper does not directly deal with reliability estimation, it identifies the potential causes of service disruption and presents the possible recommendations for the reliable operation of the communication networks. Another work has been reported in the literature that investigates the effect of communication node failures on the accuracy of the SE [53]. Using the system of systems concept, the dependency of the power system monitoring process on the communication failures has been observed. In [54], the SPCS was modeled using a queueing theoretic approach to obtain the reliability indices. Reliability was measured in terms of the probability of packet loss and communication delay. The communication link between the PMU and the PDC was modeled as a cyclic polling system and reliability parameters were obtained using Markov chains. A simultaneous optimal placement of PMUs and the PDC has been explored in [55] to enhance the reliability of data transmission. A multistage elimination method is used to obtain the optimum configuration with the highest reliability of data transmission with minimum losses. Reliability parameters were computed in [56] for the communication networks of a hierarchical WAMS using the Fault Tree Analysis (FTA). Case studies indicate that the reliability of the WAMS not only depends on the reliability of the SPCS but also on the transmission mechanism and the PDC. Assessment of Situational Awareness (SA) which is another parameter that is dependent on the reliability has been explored in [57]. Several assessment metrics are proposed and the effect of the communication system's availability on the SA has been investigated through numerous case studies. In [58], two parallel communication links have been proposed to enhance the reliability of the SPCS. One of the links is a wired link and other is a wireless link. These parallel networks can compensate for the temporary unavailability of any one of the communication link, thereby increasing the reliability of the system. In [59], Markov model (MM) based graph theory was used for assessment of the impact of communication network failures on the overall reliability of the WAMS.

\subsection{Optimal placement of Synchrophasor communication networks}

The problem of OPP is one of the widely investigated researches in the PMU parlance. As of 2018 there are over 394 research articles that have been published on this topic. Methods based on Integer Linear Programming (ILP) [60], GA [61], Particle Swarm Optimization (PSO) [62], Differential Evolution (DE) [63], simulated annealing [64], etc. have been reported in the literature. Most of these works limit themselves to finding the solution for finding the optimum locations for placement of the PMUs. They undermine the important role played by the SPCS. Consequently, very few researchers have explored the problem of the optimum placement of the SPCS and a summary of the most prominent works in this regard is presented in Table 7.

\section{Software defined networking for PMUs}

In recent years, several disruptive technologies such as IoT, 5G communications, etc., are being employed for SG applications. One such promising technology is the software-defined networking (SDN) [65]. The need for SDN arises from the limitations of the traditional communication networks. The traditional networks are rigid and are difficult to reconfigure in event of faults or other changes. Their architecture hinders the development of networking devices. The architecture of SDN is shown in Fig. 15. 
Table 7 Wide Area Damping Controllers

\begin{tabular}{|c|c|c|}
\hline Constraints & Methods used for optimal placement & Reference \\
\hline Contingency constraints on communication networks & GA & [96] \\
\hline Communication constraints on PMU placement & GA & [97] \\
\hline Data loss constraints on PMU placement & ILP & [98] \\
\hline \multirow[t]{5}{*}{ Limited communication constraints } & $\mathrm{ILP}$ & [99] \\
\hline & Multi-objective discrete Artificial Bee Colony & {$[100]$} \\
\hline & sequential quadratic programming & [101] \\
\hline & ILP & [102] \\
\hline & ILP & [103] \\
\hline Constraint on routing paths between PMUs and PDC & GA & [104] \\
\hline \multirow[t]{2}{*}{ Constraint on power system observability with maximum communication reliability } & $\mathrm{ILP}$ & [105] \\
\hline & Multi-objective GA & [106] \\
\hline Communication feasibility constraint using a minimum number of nodes. & Randomized greedy algorithm & [107] \\
\hline \multirow[t]{3}{*}{ Minimization of communication system cost } & Simulated Annealing (SA) & [108] \\
\hline & binary imperialistic competition algorithm & [109] \\
\hline & GA & {$[110,111]$} \\
\hline Constraint on communication system reliability incorporating network failures & ILP & [112] \\
\hline $\begin{array}{l}\text { Constraint on power system observability with minimum communication system } \\
\text { cost and maximum communication redundancy }\end{array}$ & Non-domination Sorting GA (NSGA-II) & [113] \\
\hline Minimization of propagation delay & Multi-objective GA & [114] \\
\hline Minimization of risk & ILP & [115] \\
\hline
\end{tabular}

In this architecture, the forwarding devices or the networking devices act according to the instructions given by the controller. The controller dynamically changes the instructions in accordance with the current requirements of the application and so is more flexible than its traditional counterpart. Thus, it simplifies the networking infrastructure management, supports new services and can cater to the demands of the envisioned exponential growth in traffic [66, 67]. The immense

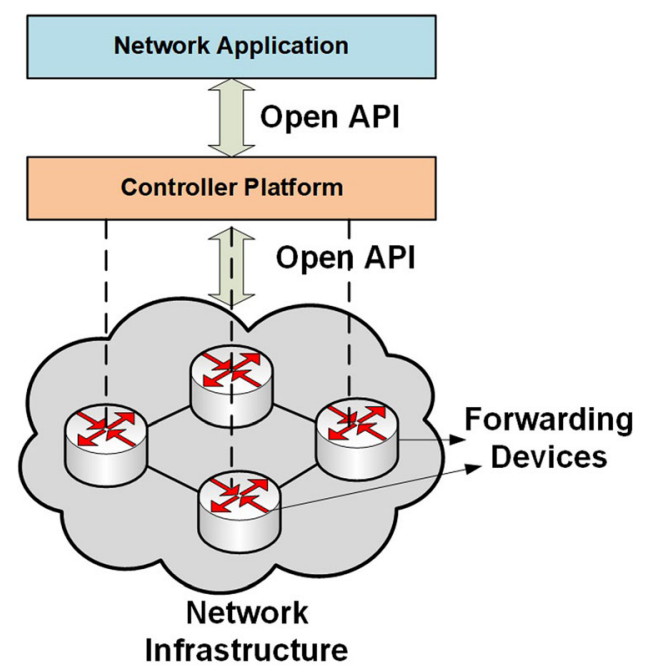

Fig. 15 SDN Architecture popularity of the SDN is its ability to address the challenges presented by the SG. There are around 120 research articles published on the topic of SDN for SGs. In SG SDN is primarily used for substation communication $[68,69]$ and the concept of SDN for PMUs is relatively less explored (5\% of the total articles published on SDN for SGs). In [70], SDN architecture was used for PMUs which makes it easier to manage the PMU traffic as compared to the IP network architecture. The state estimation from PMU data using SDN has been carried out in [67]. Recently, a PMU communication network that is resilient to cyber-attacks has been proposed in [71] that uses SDN architecture for its resiliency.

\section{Conclusion}

SPCS is an important component of the WAMS that has not been extensively explored by the power system engineers. From the review of the state-of-the-art existing knowledge gaps and potential topics for future can be identified. Communication requirements of most of the practical power grids are yet to be investigated. Co-simulation software can be used to carry out the power and communication simulations simultaneously and to obtain the characteristics of the grid. Another interesting topic of research in this context is the characterization of communication requirements of micro-grids. 
Several communication schemes have already been proposed for different synchrophasor applications. A potential topic can be the development of novel transport layer protocols for real-time implementation that is directed towards minimizing the propagation delay and packet losses. Such a research can be of immense contribution to the power grid. Another unexplored domain is the development of communication schemes for closely located micro-grids.

The other potential research topics are the development of models for estimating the reliability of the SPCS and the optimal placement of the communication infrastructure. Carrying out the reliability analysis would help in identifying the weakest component in the network, thereby incorporating sufficient component redundancy to minimize the frequency of failures. Development of models for estimating the communication infrastructure particularly using the microwave and optical fiber technology can provide valuable insights regarding the performance of these networks. Co-optimal placement of PMUs along with communication equipment can provide pragmatic results with real-world application. SDN for PMUs is yet to be fully explored by the researchers and PMU applications such as damping controllers, state estimation, etc., developed based on the SDN architecture can be of practical importance for system engineers.

\begin{abstract}
Abbreviations
CAN: Controller Area Network; CPU: Central Processing Unit; DE: Differential Evolution; DFT: Discrete Fourier Transform; DoE: Department of Energy; EDGE: Enhanced Data rates for GSM Evolution; FACTS: Flexible AC Transmission System; GA: Genetic Algorithm; GIS: Geographical Information System; GPC: Generalized Predictive Control; GPRS: General Packet Radio Service; GPS: Global Positioning System; IEEE: Institute of Electrical and Electronic Engineers; ILP: Integer Linear Programming; IoT: Internet of Things; LTEA: Long Term Evolution-Advanced; MPI: Message Passing Interface; NERC: North American Electric Reliability Corporation; NSGA-II: Non-dominating Sorting GA; OPP: Optimal Placement of PMUs; PDC: Phasor Data Concentrator; PLC: Power Line Communication; PMU: Phasor Measurement Unit; PSO: Particle Swarm Optimization; RF: Radio Frequency; ROCOF: Rate of Change of Frequency; SA: Situational Awareness; SCADA: Supervisory Control and Data Acquisition; SE: State Estimation; SIPS: System Integration Protection Schemes; SNR: Signal to Noise Ratio; SPCS: Synchro-Phasor Communication System; TVE: Total Error Vector; UMTS: Universal Mobile Telecommunications System; WAMS: Wide Area Measurement System; WLAN: Wireless Local Area Networks
\end{abstract}

\section{Acknowledgments}

Not applicable.

\section{Funding}

There are no sources of funding for the current research.

\section{Availability of data and materials}

Data sharing not applicable to this article as no datasets were generated or analyzed during the current study.

\section{Authors' contributions}

The author Mr. BA has collected the information pertaining to the state-of-theart in the synchrophasor communication system. He also prepared a crude draft of the manuscript. The author Dr. DKM provided the idea and other technical guidance required for completion of the study. He also prepared the final draft of the manuscript. Both authors have read and approved the final manuscript.

\section{Competing interests}

The authors declare that they have no competing interests.

\section{Author details}

${ }^{1}$ School of Electronics Engineering, KIIT University, Bhubaneswar 751024, India. ${ }^{2}$ Department Electrical and Electronics Engineering, Birla Institute of Technology, Mesra 835215, India.

Received: 6 July 2018 Accepted: 5 November 2018

Published online: 03 December 2018

\section{References}

1. Pourbeik, P., Kundur, P. S., \& Taylor, C. W. (2006). The anatomy of a power grid blackout - root causes and dynamics of recent major blackouts. IEEE Power and Energy Magazine, 4(5), 22-29.

2. Phadke, A. G. (2008). The wide world of wide-area measurement. IEEE Power and Energy Magazine, 6(5), 52-65.

3. Phadke, A. G., \& Thorp, J. S. (2008). Synchronized phasor measurements and their applications. New York: Springer.

4. Report of the Task Force on Communication System in Power Sector. (2016) New Delhi: Central Electricity Regulation Commission. http://www.cercind. gov.in/2016/whatsnew/RTF.pdf.

5. Mohanta, D. K., Cherukuri, M., \& Roy, D. S. (2016). A brief review of phasor measurement units as sensors for smart grid. Electric Power Components \& Systems, 44(4), 411-425.

6. Murthy, C., Varma, K. A., Roy, D. S., \& Mohanta, D. K. (2014). Reliability evaluation of phasor measurement unit using Type-2 fuzzy set theory. IEEE Systems Journal, 8(4), 1302-1309.

7. Zurawski, R. (2007). From wireline to wireless networks and technologies. IEEE Transactions on Industrial Informatics, 3(2), 93-94.

8. Deng, Y., Lin, H., Phadke, A. G., Shukla, S., \& Thorp, J. S. (2012). Networking technologies for wide-area measurement applications. In E. Hossain, Z. Han, \& H. V. Poor (Eds.), Smart Grid Communications and Networking (pp. 205-233). Cambridge: Cambridge University Press.

9. Khan, R. H., \& Khan, J. Y. (2013). A comprehensive review of the application characteristics and traffic requirements of a smart grid communications network. Computer Networks, 57(3), 825-845.

10. Berger, L., Schwager, A., \& Escudero-Garzás, J. (2013). Power line Communications for Smart Grid Applications. Journal of Electrical and Computer Engineering, 2013, 1-16.

11. Gungor, V. C., Sahin, D., Kocak, T., Ergut, S., Buccella, C., Cecati, C., \& Hancke, G. P. (2011). Smart grid technologies: Communication technologies and standards. IEEE Transactions on Industrial Informatics, 7(4), 529-539.

12. Naduvathuparambil, B., Valenti, M. C., \& Feliachi, A. (2002). Communication delays in wide area measurement systems. In Proceedings of the Thirty-Fourth Southeastern Symposium on System Theory (Cat. No.02EX540) (pp. 118-122).

13. IEEE Std 1344TM-1995. IEEE Standard for Synchrophasors for Power Systems.

14. IEEE Std C37.118TM-2005. IEEE Standard for Synchrophasors for Power Systems.

15. IEEE Std C37.118.1-2011 (Revision of IEEE Std C37.118-2005). IEEE Standard for Synchrophasor Measurements for Power Systems.

16. Martin, K. E., et al. (2015). Synchrophasor measurements under the IEEE standard C37.118.1-2011 with amendment C37.118.1a. IEEE Transactions on Power Delivery, 30(3), 1514-1522.

17. IEEE Std C37.118.2-2011 (Revision of IEEE Std C37.118-2005). IEEE Standard for Synchrophasor Data Transfer for Power Systems.

18. Martin, K. E., et al. (2014). An overview of the IEEE standard C37.118.2 Synchrophasor data transfer for power systems. IEEE Transactions on Smart Grid, 5(4), 1980-1984.

19. Phadke, A. G., \& Thorp, J. S. (2010). Communication needs for wide area measurement applications. In 5th International Conference on Critical Infrastructure, CRIS 2010 - Proceedings.

20. Lin, H., Sambamoorthy, S., Shukla, S., Thorp, J., \& Mili, L. (2012). A study of communication and power system infrastructure interdependence on PMU-based wide area monitoring and protection. In IEEE Power and Energy Society General Meeting.

21. Yang, B., Wei, L., Zhan, Z., Li, Y., Jiang, Q., \& Qi, J. (2015). Analysis on the characteristics of communication delay in wide area measuring system based on probability distribution. Dianli Xitong Zidonghua/Automation of Electric Power Systems, 39(12), 38-43 and 55.

22. Nordström, L., Chenine, M., Zhu, K., \& Vanfretti, L. (2011). Information and communication system architectures for wide-area monitoring and control applications. In 17th Power Systems Computation Conference, PSCC 2011. 
23. Chenine, M., \& Nordström, L. (2010). Performance considerations in Wide Area Monitoring and Control Systems. In 43rd International Conference on Large High Voltage Electric Systems, CIGRE 2010.

24. Chenine, M., Zhu, K., \& Nordström, L. (2009). Survey on priorities and communication requirements for PMU-based applications in the nordic region. In 2009 IEEE Bucharest PowerTech: Innovative ideas toward the electrical grid of the future.

25. Pignati, M., et al. (2015). Real-time state estimation of the EPFL-campus medium-voltage grid by using PMUs. In IEEE Power and Energy Society Innovative Smart Grid Technologies Conference, ISGT 2015.

26. Thomas, M. S., Senroy, N., \& Rana, A. S. (2014). Analysis of time delay in a wide-area communication network. In Proceedings of 6th IEEE Power India International Conference, PIICON 2014.

27. Babazadeh, D., Chenine, M., Zhu, K., Nordstrom, L., \& Al-Hammouri, A. (2013). A platform for wide area monitoring and control system ICT analysis and development. In IEEE Grenoble Conference PowerTech, POWERTECH 2013.

28. Zhu, K., Chenine, M., Nordström, L., Holmström, S., \& Ericsson, G. (2013). An empirical study of synchrophasor communication delay in a utility TCP/IP network. International Journal of Emerging Electric Power Systems, 14(4), 341-350.

29. Chenine, M., Al Khatib, I., Ivanovski, J., Maden, V., \& Nordström, L. (2010). PMU traffic shaping in IP-based wide area communication. In 5th International Conference on Critical Infrastructure, CRIS 2010 - Proceedings.

30. Chuang, C.-L., Jiang, J.-A., Wang, Y.-C., Chen, C.-P., \& Hsiao, Y.-T. (2007). An adaptive PMU-based fault location estimation system with a fault-tolerance and load-balancing communication network. In IEEE Lausanne POWERTECH, Proceedings (pp. 1197-1202).

31. Radovanovic, A. (2001). Using the internet in networking of synchronized phasor measurement units. International Journal of Electrical Power \& Energy Systems, 23(3), 245-250.

32. Ji, K., Wang, K.-Y., \& Cai, Z.-X. (2005). Communication scheme of phasor measurement unit in WAMS. Dianli Xitong Zidonghua/Automation of Electric Power Systems, 29(3), 77-80.

33. Rajput, V. S., Rovnyak, S. M., Koskie, S., \& Sheng, Y. (2008). A microcontroller-based phasor measurement system with CAN bus communication. In IEEE Power and Energy Society 2008 General Meeting: Conversion and Delivery of Electrical Energy in the 21st Century, PES.

34. Asprou, M., Kyriakides, E., Dumitrescu, A.-M., \& Albu, M. (2016). The impact of PMU measurement delays and a heterogenous communication network on a linear state estimator. In Proceedings of the 18th Mediterranean Electrotechnical Conference: Intelligent and Efficient Technologies and Services for the Citizen, MELECON 2016.

35. Balasubramaniam, K., Luitel, B., \& Venayagamoorthy, G. K. (2012). A scalable wide area monitoring system using cellular neural networks. In Proceedings of the International Joint Conference on Neural Networks.

36. Awad, A., Moarrab, S., \& German, R. (2015). QoS implementation inside LTE networks to support time-critical smart grid applications. In IEEE 15th International Conference on Environment and Electrical Engineering, EEEIC 2015 - Conference Proceedings (pp. 1204-1209)

37. Yan, D.-J., Ju, P., \& Yuan, H. (2004). Wide area measurement and real-time transmission system for phasor data under network communication mode. Power System Technology, 28(4), 15-18.

38. Xu, T., Yin, X., You, D., Li, Y., \& Wang, Y. (2008). A novel communication network for three-level wide area protection system. In IEEE Power and Energy Society 2008 General Meeting: Conversion and Delivery of Electrical Energy in the 21st Century, PES.

39. Wang, Z. J., \& Wang, Y. W. (2014). Research on the anti-grade trip system in coal mine high-voltage grid based on WAMS. In Information Technology and Computer Application Engineering - Proceedings of the 2013 International Conference on Information Technology and Computer Application Engineering, ITCAE 2013 (pp. 695-697)

40. Meloni, A., Pegoraro, P. A., Atzori, L., Castello, P., \& Sulis, S. (2016). loT cloud-based distribution system state estimation: Virtual objects and context-awareness. In IEEE International Conference on Communications, ICC 2016

41. Deng, Y., Lin, H., Phadke, A. G., Shukla, S., Thorp, J. S., \& Mili, L. (2012). Communication network modeling and simulation for wide area measurement applications. In IEEE PES Innovative Smart Grid Technologies, ISGT 2012.

42. Xin, Y., \& Chakrabortty, A. (2013). A study on group communication in distributed wide-area measurement system networks in large power systems. In IEEE Global Conference on Signal and Information Processing, GlobalSIP 2013 - Proceedings (pp. 543-546).
43. Korres, G. N., Tzavellas, A., \& Galinas, E. (2013). A distributed implementation of multi-area power system state estimation on a cluster of computers. Electric Power Systems Research, 102, 20-32.

44. Yan, C., Wu, J., \& Yang, Q. (2004). Investigation on the distributed dynamic phasor monitoring system. Dianli Xitong Zidonghua/Automation of Electric Power Systems, 28(18), 50-53.

45. Nabavi, S., Zhang, J., \& Chakrabortty, A. (2015). Distributed optimization algorithms for wide-area oscillation monitoring in power systems using interregional PMU-PDC architectures. IEEE Transactions on Smart Grid, 6(5), 2529-2538.

46. Chenine, M., \& Nordstrom, L. (2011). Modeling and simulation of wide-area communication for centralized PMU-based applications. IEEE Transactions on Power Delivery, 26(3), 1372-1380.

47. Chenine, M., \& Nordström, L. (2009). Investigation of communication delays and data incompleteness in multi-PMU Wide Area Monitoring and Control Systems. In International Conference on Electric Power and Energy Conversion Systems, (EPECS) (pp. 1-6). Sharjah: IEEE.

48. Georg, H., Wietfeld, C., Muller, S. C., \& Rehtanz, C. (2012). A HLA based simulator architecture for co-simulating ICT based power system control and protection systems. In IEEE 3rd International Conference on Smart Grid Communications, SmartGridComm 2012 (pp. 264-269).

49. Adewole, A. C., \& Tzoneva, R. (2017). Co-simulation platform for integrated real-time power system emulation and wide area communication. IET Generation, Transmission and Distribution, 11(12), 3019-3029.

50. Liu, W.-X., Liu, N., Fan, Y.-F., Zhang, L.-X., \& Zhang, X. (2009). Reliability analysis of wide area measurement system based on the centralized distributed model. In IEEE/PES Power Systems Conference and Exposition, PSCE 2009.

51. Zhao, X., Lu, J., Wang, Y., Peng, J., He, F., \& Wei, H. (2009). Reliability assessment of WAMS based on a combined hardware and software probability model of phasor measurement units. Dianli Xitong Zidonghual Automation of Electric Power Systems, 33(16), 19-23.

52. Goutard, E., Rudolph, T., \& Mesbah, M. (2010). Impact of communication network impairments on Wide Area Monitoring, Control and Protection applications in the IEC61850 environment. In 43rd International Conference on Large High Voltage Electric Systems 2010, CIGRE 2010.

53. Asprou, M., Hadjiantonis, A. M., Ciornei, I., Milis, G., \& Kyriakides, E. (2012). On the complexities of interdependent infrastructures for wide area monitoring systems. In IEEE Workshop on Complexity in Engineering, COMPENG 2012 Proceedings (pp. 1-6).

54. Menike, S., Yahampath, P., Rajapakse, A., \& Alfa, A. (2013). Queuing-theoretic modeling of a PMU communication network. In IEEE Power and Energy Society General Meeting.

55. Fesharaki, F. H., Hooshmand, R. A., \& Khodabakhshian, A. (2013). A new method for simultaneous optimal placement of PMUs and PDCs for maximizing data transmission reliability along with providing the power system observability. Electric Power Systems Research, 100, 43-54.

56. Li, J., Zhang, A., Zhang, H., Liu, X., Geng, Y., \& Wei, Y. (2015). Reliability evaluation of the wide area protect system. Diangong Jishu Xuebao/ Transactions of China Electrotechnical Society, 30(12), 344-350.

57. Sodhi, R., \& Sharieff, M. I. (2015). Phasor measurement unit placement framework for enhanced wide-area situational awareness. IET Generation, Transmission and Distribution, 9(2), 172-182.

58. Castello, P., Ferrari, P., Flammini, A., Muscas, C., Pegoraro, P. A., \& Rinaldi, S. (2015). A distributed PMU for electrical substations with wireless redundant process bus. IEEE Transactions on Instrumentation and Measurement, 64(5), 1149-1157.

59. Rana, A. S., Thomas, M. S., \& Senroy, N. (2017). Reliability evaluation of WAMS using Markov-based graph theory approach. IET Generation, Transmission and Distribution, 11(11), 2930-2937.

60. Gou, B. (2008). Optimal placement of PMUs by integer linear programming IEEE Transactions on Power Systems, 283(3), 1525-1526.

61. Aminifar, F., Lucas, C., Khodaei, A., \& Fotuhi-Firuzabad, M. (2009). Optimal placement of phasor measurement units using immunity genetic algorithm IEEE Transactions on Power Delivery, 24(3), 1014-1020.

62. Hajian, M., Ranjbar, A. M., Amraee, T., \& Mozafari, B. (2011). Optimal placement of PMUs to maintain network observability using a modified BPSO algorithm. International Journal of Electrical Power \& Energy Systems, 33(1), 28-34.

63. Peng, C., Sun, H., \& Guo, J. (2010). Multi-objective optimal PMU placement using a non-dominated sorting differential evolution algorithm. International Journal of Electrical Power \& Energy Systems, 32(8), 886-892. 
64. More, K. K., \& Jadhav, H. T. (2013). A literature review on optimal placement of phasor measurement units. In IEEE International Conference on Power, Energy and Control (ICPEC).

65. Dong, X., Lin, H., Tan, R., Iyer, R. K., \& Kalbarczyk, Z. (2015). Software-defined networking for smart grid resilience: Opportunities and challenges. In Proc. 1st ACM Workshop Cyber Phys. Syst. Security (CPSS) (pp. 61-68).

66. Kanizo, Y., Hay, D., \& Keslassy, I. (2013). Palette: Distributing tables in software-defined networks. In Proc. IEEE Int. Conf. Comput. Commun. (INFOCOM) (pp. 545-549).

67. Meloni, A., et al. (2017). Bandwidth and accuracy-aware state estimation for smart grids using software defined networks. Energies, 10(7), 858-878.

68. Molina, E., et al. (2015). Using software defined networking to manage and control IEC 61850-based systems. Computers and Electrical Engineering, 43(1), 142-154.

69. Cahn, A., et al. (2013). Software-defined energy communication networks: From substation automation to future smart grids. In Proceedings of the 2013 IEEE International Conference on Smart Grid Communications (SmartGridComm) (pp. 558-563). Vancouver: IEEE.

70. Goodney, A., Kumar, S., Ravi, A., \& Cho, Y. H. (2013). Efficient PMU networking with software defined networks. In Proc. IEEE Int. Conf. Smart Grid Commun. (SmartGridComm) (pp. 378-383).

71. Lin, H., et al. (2018). Self-healing attack-resilient PMU network for power system operation. IEEE Transactions on Smart Grid, 9(3), 1551-1565.

72. Golshani, M., Taylor, G. A., Pisica, I., \& Ashton, P. M. (2015). Performance evaluation of MPLS-enabled communications infrastructure for wide area monitoring systems. IET Seminar Digest, 2015, 1-7.

73. Chuang, C.-L., Wang, Y.-C., Lee, C.-H., Liu, M.-Y., Hsiao, Y.-T., \& Jiang, J.-A. (2010). An adaptive routing algorithm over packet switching networks for operation monitoring of power transmission systems. IEEE Transactions on Power Delivery, 25(2), 882-890.

74. Best, R. J., Morrow, D. J., Laverty, D. M., \& Crossley, P. A. (2010). Synchrophasor broadcast over internet protocol for distributed generator synchronization. IEEE Transactions on Power Delivery, 25(4), 2835-2841.

75. Myrda, P. T., \& Koellner, K. (2010). NASPInet - the internet for synchrophasors. In Proceedings of the Annual Hawaii International Conference on System Sciences.

76. Bhor, D., Angappan, K., \& Sivalingam, K. M. (2014). A co-simulation framework for Smart Grid wide-area monitoring networks. In 6th International Conference on Communication Systems and Networks, COMSNETS 2014.

77. Bhor, D., Angappan, K., \& Sivalingam, K. M. (2016). Network and power-grid co-simulation framework for Smart Grid wide-area monitoring networks. Journal of Network and Computer Applications, 59, 274-284.

78. Cherukuri, N., \& Nahrstedt, K. (2011). Cooperative congestion control in power grid communication networks. In IEEE International Conference on Smart Grid Communications, SmartGridComm 2011 (pp. 587-592).

79. Qiu, M., Su, H., Chen, M., Ming, Z., \& Yang, L. T. (2012). Balance of security strength and energy for a PMU monitoring system in smart grid. IEEE Communications Magazine, 50(5), 142-149.

80. Ambrosie, S., \& Lupu, E. C. (2014). Measurements and data communication for a small hydropower plant connected to the national grid. UPB Scientific Bulletin, Series C: Electrical Engineering and Computer Science, 76(4), 85-96.

81. Kim, D.-Y., \& Kim, Y.-C. (2015). Design and performance evaluation of hierarchical communication network for wide area measurement system. In International Conference on Smart Energy Grid Engineering, SEGE 2015.

82. Gharavi, H., \& Hu, B. (2015). Scalable Synchrophasors communication network design and implementation for real-time distributed generation grid. IEEE Transactions on Smart Grid, 6(5), 2539-2550.

83. Zhu, K., Chenine, M., Nordström, L., Holmström, S., \& Ericsson, G. (2014). Design requirements of wide-area damping systems - using empirical data from a utility IP network. IEEE Transactions on Smart Grid, 5(2), 829-838.

84. Xiaoyang, T., Guodong, L., Xiaoru, W., \& Shan, Z. (2005). The analysis of communication architecture and control mode of wide area power systems control. In Proceedings - 2005 International symposium on autonomous decentralized systems, ISADS 2005 (Vol. 2005, pp. 59-65).

85. Anh, N. T., Vanfretti, L., Driesen, J., \& Van Hertem, D. (2015). A quantitative method to determine ICT delay requirements for wide-area power system damping controllers. IEEE Transactions on Power Systems, 30(4), 2023-2030.

86. G.-L. Yu, B.-H. Zhang, H. Xie, C.-G. Wang, B.-G. Zou, and D.-P. Wang, "Design of a nonlinear robust integrated controller based on incomplete time-delay wide-area measurement information," Zhongguo Dianji Gongcheng Xuebao/Proceedings of the Chinese Society of Electrical Engineering, vol. 27, no. 10. pp. 7-13, 2007.
87. Huaren, W., Qi, W., \& Xiaohui, L. (2008). PMU-based wide area damping control of power systems. In Joint International Conference on Power System Technology POWERCON and IEEE Power India Conference, POWERCON 2008.

88. Fan, L. (2008). Synchronized global Phasor Measurement based inter-area oscillation control considering communication delay. In IEEE Power and Energy Society 2008 General Meeting: Conversion and Delivery of Electrical Energy in the 21st Century, PES.

89. Saejia, M., \& Ngamroo, I. (2010). Wide area robust TCSC controller design considering communication delay uncertainty. In ECTI-CON 2010 - The 2010 ECTI International Conference on Electrical Engineering/Electronics, Computer, Telecommunications and Information Technology (pp. 996-1000).

90. Zhu, K., \& Nordström, L. (2014). Design of wide-area damping systems based on the capabilities of the supporting information communication technology infrastructure. IET Generation, Transmission and Distribution, 8(4), 640-650.

91. Chaudhuri, N. R., Chakraborty, D., \& Chaudhuri, B. (2011). An architecture for FACTS controllers to deal with bandwidth-constrained communication. IEEE Transactions on Power Delivery, 26(1), 188-196.

92. Liu, J., Gusrialdi, A., Hirche, S., \& Monti, A. (2011). Joint controller-communication topology design for distributed wide-area damping control of power systems. IFAC Proceedings Volumes (IFAC-PapersOnline), 18, 519-525.

93. Zhu, K., Rahimi, S., Nordström, L., \& Zhang, B. (2015). Design phasor data concentrator as adaptive delay buffer for wide-area damping control. Electric Power Systems Research, 127, 22-31.

94. Wang, Y., Yemula, P., \& Bose, A. (2015). Decentralized communication and control systems for power system operation. IEEE Transactions on Smart Grid, 6(2), 885-893.

95. Singh, V. P., Kishor, N., \& Samuel, P. (2016). Communication time delay estimation for load frequency control in two-area power system. Ad Hoc Networks, 41, 69-85.

96. Aminifar, F., Khodaei, A., Fotuhi-Firuzabad, M., \& Shahidehpour, M. (2010). Contingency-constrained PMU placement in power networks. IEEE Transactions on Power Systems, 25(1), 516-523.

97. Miljanic, Z., Djurovic, I., \& Vujoševic, I. (2013). Multiple channel PMU placement considering communication constraints. Energy Systems, 4(2), 125-135.

98. Tai, X., Marelli, D., Rohr, E., \& Fu, M. (2011). Optimal PMU placement for power system state estimation with random communication packet losses. In IEEE International Conference on Control and Automation, ICCA (pp. 444-448).

99. Anees, M. A., \& Rihan, M. (2014). Optimal placement of phasor measurement units considering communication unavailability. In IEEE International Workshop on Applied Measurements for Power Systems, AMPS 2014 Proceedings (pp. 75-80).

100. Mahapatra, K., Nayak, M. R., \& Rout, P. K. (2015). Multi-objective discrete artificial bee Colony based phasor measurement unit placement for complete and incomplete observability analysis (Vol. 308). Bhubaneswar: AISC.

101. Theodorakatos, N. P., Manousakis, N. M., \& Korres, G. N. (2015). A sequential quadratic programming method for contingency-constrained phasor measurement unit placement. International Transactions on Electrical Energy Systems, 25(12), 3185-3211.

102. Theodorakatos, N. P., Manousakis, N. M., \& Korres, G. N. (2015). Optimal placement of phasor measurement units with linear and non-linear models. Electric Power Components \& Systems, 43(4), 357-373.

103. Gaber, A., Seddik, K. G., \& Elezabi, A. Y. (2016). PMUs placement with max-flow min-cut communication constraint in smart grids. In IEEE Wireless Communications and Networking Conference, WCNC (Vol. 2016-September).

104. Shahraeini, M., Javidi, M. H., \& Ghazizadeh, M. S. (2013). Communication infrastructure planning for wide area measurement systems in power systems. International Journal of Communication Networks and Distributed Systems, 10(4), 319-334.

105. Haghighatdar Fesharaki, F., Hooshmand, R.-A., \& Khodabakhshian, A. (2014). Simultaneous optimal design of measurement and communication infrastructures in hierarchical structured WAMS. IEEE Transactions on Smart Grid, 5(1), 312-319.

106. Appasani, B., \& Mohanta, D. K. (2018). A two-stage Markov model aided frequency duration approach for reliability analysis of PMU microwave communication networks. In Proceedings of the Institution of Mechanical Engineers, Part O: Journal of Risk and Reliability. https://doi.org/10.1177/ $1748006 \times 18785685$

107. Ghosh, D., Ghose, T., \& Mohanta, D. K. (2013). Communication feasibility analysis for smart grid with phasor measurement units. IEEE Transactions on Industrial Informatics, 9(3), 1486-1496. 
108. Georges, D. (2014). Optimal PMU-based monitoring architecture design for power systems. Control Engineering Practice, 30, 150-159.

109. Mohammadi, M. B., Hooshmand, R.-A., \& Fesharaki, F. H. (2016). A new approach for optimal placement of PMUs and their required communication infrastructure in order to minimize the cost of the WAMS. IEEE Transactions on Smart Grid, 7(1), 84-93.

110. Shahraeini, M., Ghazizadeh, M. S., \& Javidi, M. H. (2012). Co-optimal placement of measurement devices and their related communication infrastructure in wide area measurement systems. IEEE Transactions on Smart Grid, 3(2), 684-691.

111. Singh, S. P., \& Singh, S. P. (2017). Optimal cost wide area measurement system incorporating communication infrastructure. IET Generation, Transmission and Distribution, 11(11), 2814-2821.

112. Sarailoo, M., \& Wu, N. E. (2016). A new PMU placement algorithm to meet a specified synchrophasor availability. In IEEE Power and Energy Society Innovative Smart Grid Technologies Conference, ISGT 2016.

113. Basetti, V., \& Chandel, A. K. (2016). Simultaneous placement of PMUs and communication infrastructure in WAMS using NSGA-II. IETE Technical Review, 33(6), 621-637.

114. Appasani, B., \& Mohanta, D. K. (2018). Co-optimal placement of PMUs and their communication infrastructure for minimization of propagation delay in the WAMS. IEEE Transactions on Industrial Informatics, 14(5), 2120-2132.

115. Appasani, B., \& Mohanta, D. K. (2017). Optimal placement of synchrophasor sensors for risk hedging in a smart grid. IEEE Sensors Journal, 17(23), 7857-7865.

\section{Submit your manuscript to a SpringerOpen ${ }^{\circ}$ journal and benefit from:}

- Convenient online submission

- Rigorous peer review

- Open access: articles freely available online

High visibility within the field

- Retaining the copyright to your article

Submit your next manuscript at $\boldsymbol{\nabla}$ springeropen.com 